\title{
Heart-rate independent myocardial T1-mapping using combined saturation and inversion preparation pulses
}

\author{
Sebastian Weingärtner ${ }^{1,3^{*}}$, Mehmet Akcakaya ${ }^{1}$, Sophie Berg ${ }^{1}$, Kraig V Kissinger ${ }^{1}$, Warren J Manning ${ }^{1,2}$, Reza Nezafat $^{1}$ \\ From 16th Annual SCMR Scientific Sessions \\ San Francisco, CA, USA. 31 January - 3 February 2013
}

\section{Background}

Myocardial T1 mapping remains a challenging task due to restrictions imposed by cardiac and respiratory motion. Modified Look-Locker Inversion Recovery (MOLLI) [1] is widely used for 2D cardiac T1-mapping. In MOLLI, the spin-lattice relaxation curve is sampled several times after a single magnetization preparation. The ECG triggered imaging induces a disturbance in the relaxation curve, which varies based on the heart rate. Hence, MOLLI T1 measurements show strong correlations to the heart rate especially in pre-contrast. We developed a novel T1 mapping sequence that enables heart-rate invariant myocardial T1 mapping.

\section{Methods}

Figure 1 shows the schematic of the proposed SAturation Pulse Prepared Heart rate independent Inversion-REcovery sequence (SAPPHIRE). A saturation pulse is inserted right after the $\mathrm{R}$-wave of selected heart-cycles. This dephases the magnetization in the imaging volume and eliminates the need for recovery periods after the magnetization preparation. The saturation pulse is followed by an

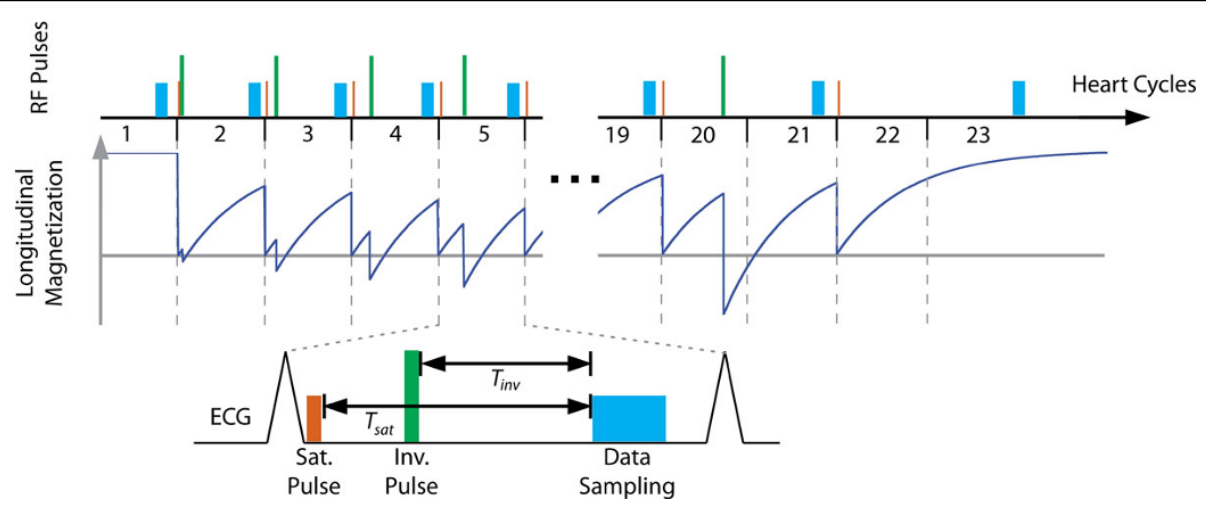

Figure 1 Sequence diagram depicting the SAPPHIRE T1-mapping sequence: a saturation pulse is performed after the R-wave to erase the magnetization history. It is followed by the inversion pulse and a single-shot image readout. To extend the range of applicable inversion times the data readout of some SAPPHIRE experiments is performed in the heart-cycle after the magnetization preparation. Additionally the first heartcycle is performed without magnetization preparation and the last heart-cycle with the saturation pulse only. This increases the effective inversion times and improves the T1 fit.

$\overline{1}$ Department of Medicine, Beth Israel Deaconess Medical Center and Harvard

Medical School, Boston, MA, USA

Full list of author information is available at the end of the article 

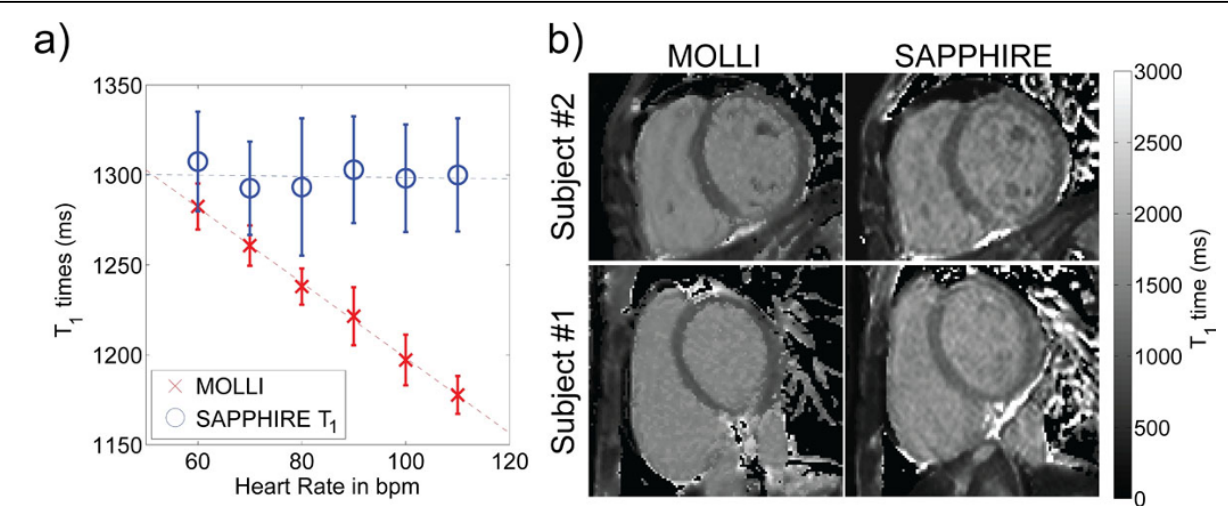

Figure 2 a) Calculated T1 values from phantom images acquired with different simulated R-R interval of 60-120 beats per minute. T1 measurements calculated from MOLLI shows linearly dependent on the duration of R-R, however SAPPHIRE T1 measurements were relatively constant. b) T1 maps from two healthy subjects acquired without gadolinium contrast showing higher homogeneity of the T1 measurements in SAPPHIRE.

inversion pulse after a variable delay to create various T1 weighted contrasts in the images. Eleven SAPPHIRE images are acquired, where each magnetization preparation is followed by a single-shot imaging in the same heart-cycle. Six additional SAPPHIRE images are acquired with longer inversion times, by performing the data sampling in the heart-cycle after the magnetization preparation. The first heart cycle is performed without any prepulses, to provide a spin-density weighted image, which facilitates the T1-fit.

SAPPHIRE T1-mapping was compared to MOLLI in phantom measurements and in healthy volunteers. A bottle phantom with a T1 of $\sim 1300 \mathrm{~ms}$ was imaged using both T1-mapping sequences at various simulated ECGs with different heart-rates. Furthermore, pre-contrast T1-maps in five healthy volunteers were acquired using SAPPHIRE T1-mapping and MOLLI.

\section{Results}

In the phantom measurements SAPPHIRE T1-mapping is in good agreement with MOLLI measurements at a simulated heart-rate of $60 \mathrm{bpm}$ (Relative difference: $<2 \%$ ). The SAPPHIRE T1-times, as depicted in Figure 2a), showed no significant correlation with the heart rate $(r=-0.10)$, while MOLLI is highly correlated $(r=-0.99)$. The T1 times in myocardium and the blood pool of the LV of the volunteers showed no significant difference between the two sequences $(p=0.20, p=0.10)$. Figure $2 b)$ shows exemplary T1-maps of two subjects. SAPPHIRE T1-mapping required slightly longer breath holds (16-23s SAPPHIRE vs. 12-17s MOLLI).

\section{Conclusions}

SAPPHIRE T1-mapping enables heart rate independent myocardial T1-mapping. The heart-rate invariance is achieved by applying a combination of saturation and inversion pulses as magnetization preparation.

\section{Funding}

Deutsche Telekom Stiftung; NIH:R01EB008743-01A2; NIH: K99HL111410-01.

\section{Author details}

'Department of Medicine, Beth Israel Deaconess Medical Center and Harvard Medical School, Boston, MA, USA. ${ }^{2}$ Department of Radiology, Beth Israel Deaconess Medical Center and Harvard Medical School, Boston, MA, USA. ${ }^{3}$ Computer Assisted Clinical Medicine, University Medical Center Mannheim, Heidelberg University, Mannheim, Germany.

Published: 30 January 2013

\section{Reference}

1. Messroghli:. MRM 2008.

doi:10.1186/1532-429X-15-S1-P46

Cite this article as: Weingärtner et al:: Heart-rate independent myocardial T1-mapping using combined saturation and inversion preparation pulses. Journal of Cardiovascular Magnetic Resonance 201315 (Suppl 1):P46.

\section{Submit your next manuscript to BioMed Central} and take full advantage of:

- Convenient online submission

- Thorough peer review

- No space constraints or color figure charges

- Immediate publication on acceptance

- Inclusion in PubMed, CAS, Scopus and Google Scholar

- Research which is freely available for redistribution
Biomed Central 